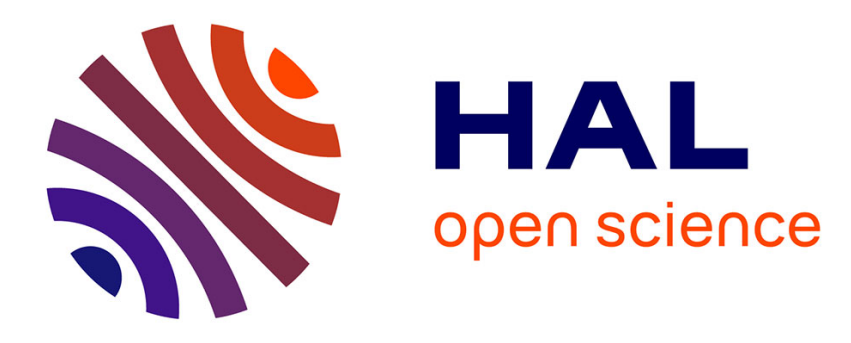

\title{
Supervised Classification of Activities of Daily Living in Health Smart Homes using SVM
}

\author{
Anthony Fleury, Norbert Noury, Michel Vacher
}

\section{To cite this version:}

Anthony Fleury, Norbert Noury, Michel Vacher. Supervised Classification of Activities of Daily Living in Health Smart Homes using SVM. IEEE EMBC 2009, Sep 2009, Minneapolis, France. pp.6099 6102. hal-00431887

\author{
HAL Id: hal-00431887 \\ https://hal.science/hal-00431887
}

Submitted on 13 Nov 2009

HAL is a multi-disciplinary open access archive for the deposit and dissemination of scientific research documents, whether they are published or not. The documents may come from teaching and research institutions in France or abroad, or from public or private research centers.
L'archive ouverte pluridisciplinaire HAL, est destinée au dépôt et à la diffusion de documents scientifiques de niveau recherche, publiés ou non, émanant des établissements d'enseignement et de recherche français ou étrangers, des laboratoires publics ou privés. 


\title{
Supervised Classification of Activities of Daily Living in Health Smart Homes using SVM
}

\author{
Anthony Fleury*, Member, IEEE, Norbert Noury, Senior Member, IEEE, and Michel Vacher
}

\begin{abstract}
By 2050, about a third of the French population will be over 65 . To face this modification of the population, the current studies of our laboratory focus on the monitoring of elderly people at home. This aims at detect, as early as possible, a loss of autonomy by objectivizing criterions such as the international ADL or the French AGGIR scales implementing automatic classification of the different Activities of Daily Living. A Health Smart Home is used to achieve this goal. This flat includes different sensors. The data from the various sensors were used to classify each temporal frame into one of the activities of daily living that has been previously learnt (seven activities: hygiene, toilets, eating, resting, sleeping, communication and dressing/undressing). This is done using Support Vector Machines. We performed an experimentation with 13 young and healthy subjects to learn the model of activities and then we tested the classification algorithm (crossvalidation) on real data.
\end{abstract}

Index Terms-Support vector machines, Activities of Daily Living, Health Smart Homes, Sensor Fusion.

\section{INTRODUCTION}

$\mathbf{T}$ HE average age of the population in the developed countries is increasingly growing due to improvements in medicine. For the year 2050, the United Nations predict $22 \%$ of people over 65 in the world. The countries have to be prepared to face this demographic modification so that elderly can live in the best possible conditions.

That is the reason why researchers work on telemedicine and telemonitoring solutions to allow elderly people to stay at home as long as possible. Lots of projects include smart homes to achieve this goal [1]. Several solutions are studied by laboratories and companies to monitor the health of people at home. These solutions include different level of complexity and technological challenges. Some projects decided to monitor only few parameters to characterize the health of the person. For instance, in France, Edelia monitors the water consumption and its possible drift to detect behavior modification and in Japan, Zojirushi Corporation is interested by the use of the electric water boiler. For these two projects,

Anthony Fleury is with the Laboratory TIMC-IMAG, UMR CNRS/UJF 5525, team AFIRM. Faculté de Médecine de Grenoble, bâtiment Jean Roget, 38706 La Tronche, France (e-mail: fleury_anthony@ hotmail.com).

Norbert Noury is University Professor at the Institute of Nanotechnologies of Lyon, INL-INSA Lyon, team MMB, F69621 Villeurbanne, France (norbert.noury@insa-lyon.fr) and associate researcher at lab. TIMC-IMAG (norbert.noury@imag.fr).

Michel Vacher is with the LIG Laboratory, UMR UJF/CNRS/INPG 5217, team GETALP. 220 rue de la Chimie, BP 53, 38041 Grenoble Cedex 9. (e-mail: Michel.Vacher@imag.fr). families can have access to the uses and behaviours of the person and will also be noticed of an important modification.

To complete this analysis and obtain a more detailed description of the autonomy of the person, we can fuse a large number of sensors distributed inside the flat to determine the activities of daily living that are performed by the elderly people. That has been begun in some projects throughout the world. The CARE project [2] uses HMM (Hidden Markov Models) with lots of sensors (localization, temperature...) in a Health Smart Home and learns to recognize correctly, after a training phase, the two activities "going to the toilets" and "exit from the flat". In another project [3], objects and foods are tagged to create a model that distinguishes the activities of preparing a drink (cold or hot) from the hygiene. Electrical signatures analysis is another path that is tested in [4], [5] to detect the different activities of daily living. In this article, we focused on the use of Support Vector Machine to learn seven different activities and classify them using sensors inside the flat that will be described in the next sections. We also performed an experimentation to learn the activities and test the classification algorithm with cross-validation.

\section{Health Smart Home for Activities of Daily LIVING RECOGNITION}

\section{A. The Grenoble Health Smart Home}

In the year 1999, the researchers of the TIMC-IMAG laboratory of Grenoble installed, inside the faculty of medicine of Grenoble, a real F2 flat, with all the rooms and the comfort required. This is composed of a bedroom, a living-room, a hall, a kitchen (with cupboards, fridge...), a bathroom with a shower and a cabinet.

This flat, in order to be used to monitor people at home, has since been equipped with several sensors:

- Presence infra-red sensors, for the location of the person inside the flat. These sensors are wirelessly linked to a computer that save, into a database, all the detection that occurs (time and detector). For this detector, each time a movement is detected in its space, a detection is sent. We can, as a consequence, have more than one following detection on a given sensor.

- Door contacts for the detection of the use of some of the commodities (cupboard, fridge and convenient).

- Microphones, to process sounds inside the flat. For sounds of daily living, the software was trained in order to recognize eight different classes (door clapping, door 


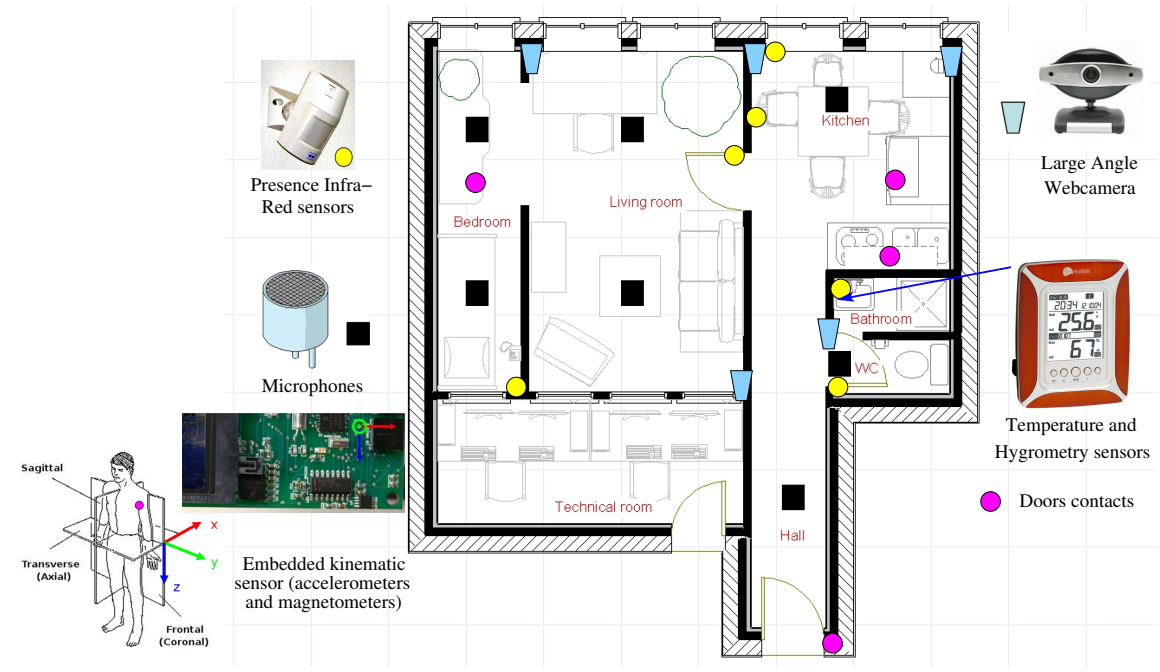

Fig. 1. Organization and sensor distribution in the Health Smart Home of the TIMC-IMAG at the faculty of Medicine of Grenoble

locking, step sound, dishes sound, phone ringing, fall of an object, glass breaking and screaming). For the speech processing, an autonomous speech recognizer was designed to recognize the five most probable uttered sentences for each given sound. The materials, software and a primary validation of this modality, performed in the Health Smart Home and compared to the results in laboratory, are presented in [6]. This publication shows that the signal to noise ratio of the Health Smart Home have to be improved to ensure a detection as suitable as the one in laboratory.

- An embedded (on the person) kinematic sensor, equipped with a tri-axis accelerometer and a tri-axis magnetometer. It allows the detection and classification of each postural transition and so the knowledge of the posture (sit, stand, lie) at each time. Moreover, it detects the walking periods. The algorithms outputs the time of the different changes of postures and the periods of walking. It is described in more details in [6].

- Large angle webcams that are placed only to timestamp the different activities of daily living, and further to annotate data.

The distribution of the sensors is shown on the Figure 1. The technical room also represented on this figure contains the 4 different computers that receive and store the whole set of information from the health smart home. The microphones need a national instrument acquisition board and the other connections use serial or usb port.

\section{B. Activities of Daily Living}

Geriatrics use Katz ADL (Activity of Daily Living) [7] scale or AGGIR in France (Autonomy Gerontology IsoResources Group) to evaluate the autonomy of the person, by asking questions to the elderly people. Based on these scales, we defined seven activities of daily living that were interesting to monitor:
- Sleeping - A bed is available in the bedroom for the subject to sleep as long as he wants.

- Preparing and having a breakfast - The fully equipped kitchen also contains the material and foods necessary to take a breakfast. The diversity allows the occupier to take whatever he wants and to prepare it in his way. Then, he can clean up the kitchen and the dishes.

- Dressing and undressing - A set of wears are available to dress and undress.

- Resting - This activity is the largest one. The inhabitant can do whatever he wants and like to do during his free time at home. He can read a book or a magazine, listen to the radio or watch the TV...

- Hygiene activities - During this activity the inhabitant is inside the bathroom and performs the normal hygiene activities. As it is difficult to ask the subjects to take a shower for a first experiment, we only asked them to wash their hands and teeth. For privacy respect, neither the bathroom nor the toilets are recorded on video. We asked the subject to close completely or partially the door whether he was respectively in the toilets or in the bathroom in order to differentiate both activities.

- Elimination (going to the toilets) - For this activity, the subject is in the toilets.

- Communication - This last activity consists in answering a phone call and have conversations. In our protocol, the subject is called five times on the phone and have to utter different real-, previously created, phone conversations which were randomly selected.

\section{Algorithms}

\section{A. Support Vector Machines}

Numerous methods are available to classify samples from training data. In our case, the weak number of available samples makes the learning process difficult for a large number of them (Bayesian, Neural Network...). For this reason, we 
decided to test the SVM (Support Vector Machines) method that seems to fit more to our problem and that can be used for training with small sets of data. Considering two classes of points, labelled -1 and 1 ; and a set of $\mathrm{N}$ vectors $\mathbf{x}_{i} \in X \subset \mathbb{R}^{d}, i \in[1 ; N]$ (d is the dimension of our input space) with their associated class $y_{i} \in\{-1 ; 1\}$. Supervised learning is the problem of inferring a function $f$ such as:

$$
f: X \subset \mathbb{R}^{d} \rightarrow\{-1 ; 1\}
$$

from a set of observations and that will correctly classify the maximum number of vectors $x_{i}$ and more important, that will correctly describe the phenomenon responsible of the separation between the two classes so that a new and unknown point will be classified into the correct class (capacity of generalization of the classifier).

Vapnik designed an algorithm, based on both ideas of linear separation and maximization of the margin between the separation and the nearest points of the training database [8]. This margin will give the maximum of "safety" for the generalization of the algorithm and its application to new points. SVM are widely used and showed a good capacity of generalization in various applications. Their construction is performed determining an hyperplane whose equation is $\langle\mathbf{w}, \mathbf{x}\rangle+w_{0}=0$ (where $\mathbf{w}$ and $w_{0}$ are the parameters of the equation of the hyperplane to determine). From this hyperplane, we construct the function $\mathrm{f}$ given by:

$$
\left\{\begin{array}{l}
\left\langle\mathbf{w}, \mathbf{x}_{\mathbf{i}}\right\rangle+w_{0}>0 \Rightarrow f=1 \\
\left\langle\mathbf{w}, \mathbf{x}_{\mathbf{i}}\right\rangle+w_{0}<0 \Rightarrow f=-1
\end{array}\right.
$$

$f$ is the output of the algorithm for a new point $x_{i}$. The hyperplane is constructed by solving the following equation that maximizes the margin:

$$
\arg \max _{w, w_{0}} \min _{i=1 . . N}\left\{\left\|x-x_{i}\right\|: x \in \mathbb{R}^{d},\langle\mathbf{w}, \mathbf{x}\rangle+w_{0}=0\right\}
$$

by solving the linear problem (using the lagrangian):

$$
\operatorname{Min} \quad \frac{1}{2}\|\mathbf{w}\|^{2} \quad \text { s.t. } f\left(\left\langle\mathbf{w}, \mathbf{x}_{\mathbf{i}}\right\rangle+w_{0}\right) \geq 1, i=1 . . N
$$

The previous equation stands for the linearly separable case but a large part of the problems are not included. In all this formulation, every equations in which the points of the training dataset appear rely only on inner product. Based on [9], some functions $\mathrm{K}$ acts as an inner products $\left(K\left(\mathbf{x}_{\mathbf{i}}, \mathbf{x}_{\mathbf{j}}\right)=\left\langle\mathbf{x}_{\mathbf{i}}, \mathbf{x}_{\mathbf{j}}\right\rangle\right)$. Such function will map the input space into a high-dimensional space (even infinite), named feature space in which the separation could be linear. The problem of determining the best kernel for a given application is an open address. The resolution is obtainned by replacing the dot products $\langle\cdot, \cdot\rangle$ in the lagrangian by the kernel $K(\cdot, \cdot)$. The construction of such a function follow the Mercer conditions. We used two generic kernels for comparison in our application:

- Polynomial kernel: $K\left(\mathbf{x}_{\mathbf{i}}, \mathbf{x}_{\mathbf{j}}\right)=\left(\mathbf{x}_{\mathbf{i}}^{T} \cdot \mathbf{x}_{\mathbf{j}}+1\right)^{p}$

- Gaussian kernel (RBF): $K\left(\mathbf{x}_{\mathbf{i}}, \mathbf{x}_{\mathbf{j}}\right)=\exp \left(-\frac{\left\|\mathbf{x}_{\mathbf{i}}-\mathbf{x}_{\mathbf{j}}\right\|^{2}}{2 \sigma^{2}}\right)$
Now, to realize Multiclass classification, three methods exist (M-SVM, one-versus-one and one-versus-all). We selected the one-versus-one method because it minimizes the indetermination zone (compared to the one-versus-all) and it does not necessitate larger training datasets to solve more complex problems. This scheme consists in constructing $\frac{N \cdot(N-1)}{2}$ classifiers, using all the pairwise combinations of the $\mathrm{N}$ classes. In this case, we will construct binary classifiers to differentiate the classes $C_{i}$ and $C_{j}, 0<i \leq N$ and $0<j<i$. To determine the class of a new point (the isolated point on the figure for instance), a majority voting is taken. Mathematically speaking, the decision is given by: $C=\max _{k=1 . . N} \operatorname{Card}\left(\left\{y_{i, j}\right\} \cap\{k\}\right)$ where $y_{i, j}$ is the decision given, for this new point, by the SVM trained to distinguish the classes $i$ and $j$. In case of equality, the class chosen is the one with the maximal margin from the final subset.

\section{B. Features Extraction}

The table I sums up the set of features selected for each sensor. These features have been selected by performing preexperimentation on two subjects and finding the smallest set of variables that describe the activities.

\begin{tabular}{|c|c|c|}
\hline Modality & Features selected & $\begin{array}{l}\text { Examples of } \\
\text { Information }\end{array}$ \\
\hline Actimeter & $\begin{array}{l}\text { Percentage of time } \\
\text { spent in the different } \\
\text { posture (stand, sit, lie) } \\
\text { and walking }\end{array}$ & $\begin{array}{l}\text { Sleeping (lying posture), } \\
\text { resting and cooking } \\
\text { (walking and sitting } \\
\text { down) }\end{array}$ \\
\hline Microphones & $\begin{array}{l}\text { Number of events } \\
\text { per class and } \\
\text { number of events } \\
\text { per microphones }\end{array}$ & $\begin{array}{l}\text { Communication (phone } \\
\text { ringing and speech), } \\
\text { cooking } \\
\text { sounds)... }\end{array}$ \\
\hline PIR & $\begin{array}{l}\text { Percentage of time in } \\
\text { each rooms and num- } \\
\text { ber of events for each } \\
\text { detectors }\end{array}$ & $\begin{array}{l}\text { Cooking (in the } \\
\text { kitchen), hygiene (in the } \\
\text { bathroom), elimination } \\
\text { (in the cabinet)... }\end{array}$ \\
\hline Door contacts & $\begin{array}{l}\text { Percentage of time } \\
\text { in "open" position } \\
\text { and predominant state } \\
\text { (open/close) in the } \\
\text { considered time slot }\end{array}$ & $\begin{array}{l}\text { Cooking (use of the cup- } \\
\text { board and fridge), dress- } \\
\text { ing/undressing (use of } \\
\text { the convenient) }\end{array}$ \\
\hline Environmental & $\begin{array}{l}\text { Differential measure } \\
\text { for the last } 15 \text { minutes } \\
\text { for temperature and } \\
\text { hygrometry }\end{array}$ & $\begin{array}{l}\text { Hygiene (use of the } \\
\text { shower) }\end{array}$ \\
\hline
\end{tabular}

TABLE I

SUM-UP OF THE DIFFERENT MODALITIES WITH SELECTED FEATURES AND THE ACTIVITY FOR WHICH IT IS INFORMATIVE

\section{EXPERIMENTATIONS IN REAL ENVIRONMENT}

\section{A. Protocol}

The experimental protocol was designed to construct a training and validation database to test the previous algorithm. For these measurements, 13 subjects ( 6 women and 7 
men), young and healthy (mean age 30.4 years $\pm 5.9,24-43$ min-max), were enrolled. They firstly visited the whole flat to be familiar with its organization and not to have to search every things they need. Then, the only requirement was that they have to perform at least one time each activity described in the previous section. They have no notice on the order, the time to spent for each activity etc. Once they consider that they have finished they have to leave the flat and the experimentation ends. While the experimentation runs, they have no contact with the supervisors. The mean duration of the experimentation was 55 minutes (23 minutes min and 1 hour 35 minutes max). Every things needed was available (the food, the TV, few books, etc.) to complete the activities.

\section{B. Data organization and validation method}

The computers are synchronized using NTP (Network Time Protocol) to have the same date and time. Then, they are manually labelled using the video records. From these videos, we constructed, for each experimentation, an XML file that describe the date and time of beginning and end of each activity. We work with fixed size time frames. These windows are of length 3 minutes (that corresponds to the mean time to correctly execute the shortest activity. Table II gives the organization of the training dataset from this experimental protocol. The validation method that has been chosen is the leave-one-out (due to the weak number of samples). Data are normalized (centered and reduced) at each execution of the algorithm. The coefficients of normalization are determined using the training database (without the tested sample) and the last sample is normalized using the same coefficients.

\section{TABLE II}

REPARTITION OF THE DIFFERENT CLASSES IN THE LEARNING DATASET AND RESULTS FOR THE POLYNOMIAL KERNEL (DEGREE=2) AND GaUsSian KeRnel (OPTIMAL VALUe of $\sigma$ )

\begin{tabular}{lccc}
\hline & & \multicolumn{2}{c}{ Classification results } \\
\cline { 3 - 4 } Class & Training size & Polynomial & Gaussian \\
\hline Sleeping & 49 & $77.55 \%$ & $93.87 \%$ \\
Resting & 75 & $76.71 \%$ & $78.08 \%$ \\
Dressing & 16 & $56.25 \%$ & $75.00 \%$ \\
Eating & 45 & $84.44 \%$ & $97.78 \%$ \\
Elimination & 16 & $68.75 \%$ & $93.75 \%$ \\
Hygiene & 14 & $50.00 \%$ & $64.28 \%$ \\
Communication & 17 & $89.47 \%$ & $89.47 \%$ \\
\hline Total & 252 & $75.86 \%$ & $86.21 \%$ \\
\hline
\end{tabular}

\section{Results, Discussion AND CONCLUSion}

Table II gives the results for both polynomial and Gaussian kernel. For these two kernels, the hyper-parameter has been optimized to minimize the global error rate. The first remark is that Gaussian Kernel (with adapted parameter) is more accurate than Polynomial one for our set of data. For this kernel, resting, dressing and hygiene are less successfully recognized. For the resting activity this is due to the large panel of activities that could be done by the subject. On the contrary to other ones, this activity is less restricted. For the two others, this can be explained by the weak number of samples in the training database giving a bad description of it. But in general, without considering the context, the global recognition rate is good and the results are promising.

These results are preliminary and we have to perform further experimentations to construct a more important database and allow to design a test database instead of using crossvalidation. It will allow us to test with other cross-validation processes and determined more accurately the influence of the kernel, of the hyper parameters and finally to have a better evaluation of the generalization of this algorithm. It will be also interesting to verify the importance of each sensor and the comportment of different learning-based algorithms. This work has already started and showed us that for the moment the posture is badly recognized comparing to the results when the sensor has been tested alone. The future of our work will also be to annotate all the changes of posture and the walking periods in the video and compare the results with these more accurate data for this modality. A more important database could also help us to construct another class, that takes into account transition between two activities (here as it is labelled, this is not a problem, but for a long term analysis without labelling we will meet transitions) and also to introduce knowledge into the process (time and location are information that can reduce the set of probable activities).

\section{ACKNOWLEDGMENT}

The authors would like to thanks all the subjects for their time spent doing the experiments.

\section{REFERENCES}

[1] M. Chan, D. Estève, C. Escriba, and E. Campo, "A review of smart homes- present state and future challenges." Computer Methods and Programs in Biomedicine, vol. 91, no. 1, pp. 55-81, Jul 2008.

[2] B. Kröse, T. van Kasteren, C. Gibson, and T. van den Dool, "Care: Context awareness in residences for elderly," in 6th International Conference of the International Society of Gerontology, Pisa, Tuscany, Italy, June 4-7 2008, p. 145 (6p).

[3] X. Hong, C. Nugent, M. Mulvenna, S. McClean, and B. Scotney, "Evidential fusion of sensor data for activity recognition in smart homes," Pervasive and Mobile Computing, vol. 5, no. 3, pp. 1-17, 2008.

[4] S. Tsukamoto, H. Hoshino, and T. Tamura, "Study on indoor activity monitoring by using electric field sensor," in 6th International Conference of the International Society of Gerontology, Pisa, Tuscany, Italy, June 4-7 2008, p. 226 (4p).

[5] M. Berenguer, M. Giordani, F. Giraud-By, and N. Noury, "Automatic detection of activities of daily living from detecting and classifying electrical events on the residential power line," in 10th IEEE Intl. Conf. on e-Health Networking, Applications and Service, 2008, pp. 29-32.

[6] A. Fleury, M. Vacher, H. Glasson, J.-F. Serignat, and N. Noury, "Data fusion in health smart home: Preliminary individual evaluation of two families of sensors," in 6th International Conference of the International Society of Gerontology, Pisa, Italy, June 4-7 2008, p. 135 (6p).

[7] S. Katz and C. Akpom, "A measure of primary sociobiological functions," Intl. Journal of Health Services, vol. 6, no. 3, pp. 493-508, 1976.

[8] B. E. Boser, I. M. Guyon, and V. N. Vapnik, "A training algorithm for optimal margin classifiers," in Fifth Annual Workshop on Computational Learning Theory, ACM, Pittsburgh, 1992, pp. 144-152.

[9] M. Aizerman, E. Braverman, and L. Rozonoer, "Theoretical foundations of the potential function method in pattern recognition learning," Automation and Remote Control, vol. 25, pp. 821-837, 1964. 